CERIAS Tech Report 2004-08

\title{
A TRUST-BASED CONTEXT-AWARE ACCESS CONTROL MODEL FOR
} WEB-SERVICES

by Rafae Bhatti, Elisa Bertino, Arif Ghafoor

Center for Education and Research in

Information Assurance and Security,

Purdue University, West Lafayette, IN 47907-2086 


\title{
$\underline{\text { A Trust-based Context-Aware Access Control Model for Web-Services }}$
}

\author{
Rafae Bhatti, Elisa Bertino, Arif Ghafoor
}

Contact author: rafae@purdue.edu

\begin{abstract}
A key challenge in Web services security is the design of effective access control schemes that can adequately meet the unique security challenges posed by the Web services paradigm. Despite the recent advances in Web based access control approaches applicable to Web services, there remain issues that impede the development of effective access control models for Web services environment. Amongst them are the lack of context-aware models for access control, and reliance on identity or capability-based access control schemes. In this paper, we motivate the design of an access control scheme that addresses these issues, and propose an extended, trust-enhanced version of our XML-based Role Based Access Control ( $X$ $R B A C)$ framework that incorporates context-based access control. We outline the configuration mechanism needed to apply our model to the Web services environment, and also describe the implementation architecture for the system.
\end{abstract}

Keywords: XML, Role-Based Access Control, Trust Management, Web-Services

\section{Introduction}

Security in Web services is critical to their wide-scale adoption and integration in Webbased enterprise systems and softwares. The present day Web is abound with examples of Web-based enterprise services, and there is an increasing trend amongst them to migrate to the Web services platform in order to enhance and diversify the online services provided to their customers. While shifting from the traditional client-server architecture to Web services technology is seen as an endorsement of the Internet community's faith in the promise of the Web services paradigm, the goals of interoperability and ubiquity as envisioned by the Web services technology can only reasonably be realized if the unique security challenges posed by this paradigm are appropriately addressed. Among these challenges is to develop models for effective access control in dynamic XML-based Web services. The uniqueness here comes from the fact that the Web-based enterprise resources being exposed via Web services are typically dynamic and distributed in nature, and hence require adaptive access control models that can capture the dynamically changing security requirements of the target enterprise.

The mechanisms required to effectively enforce access control across distributed, heterogeneous domains are becoming increasingly complex. This complexity arises not only because of the sheer size of the distributed clientele accessing online services but also because of the fact that access control model should capture security-relevant contextual information, such as time, location, or environmental state available at the time the access requests are made, and incorporate it in its access control decisions. These context parameters capture the dynamically changing access requirements in a Webbased enterprise, and hence are critical to the effectiveness of the resulting access control scheme. The context directly affects the level of trust associated with a user, and hence the authorizations granted to him/her. These parameters constitute what is generally termed as a "user profile". The access privileges of requestors to an online service provider could be based on certain thresholds as established by the System Security Officer (SSO) based on the requestor's access patterns. If at any time, a requestor appears 
to deviate from his/her usual profile, the thresholds (i.e. the trust level) would automatically be reduced as a precaution to prevent a potential abuse of privileges. This is a real-time requirement, and is exceedingly important in dynamic Web services serving millions of customers with diverse activity profiles. In order for the access control to be effectively exercised in such scenarios with context-sensitive access requirements, the traditional access control models must be extended to make them context-aware. To this end, we propose to employ the generalized temporal extension to our X-RBAC [XRBAC] model, the XML-based Generalized Temporal Role Based Access Control (XGTRBAC) model [X-GTRBAC]. X-GTRBAC was originally proposed as a solution to enterprise-wide access control, but due to its XML-based framework, it can also be configured to provide access control in Web services. In Section 3, we introduce the reader to the X-GTRBAC model and outline the mechanism to extend X-GTRBAC as a context-aware access control framework for Web services environment.

Another issue we highlight in the paper is trust-based role assignment to users. There are different (although related) notions of "trust" in the literature. The one that is relevant to our purposes is the level of confidence associated with a user based on certain certified attributes thereof. In our framework, this level of confidence is not quantitatively reported. Instead, we rely on the Trust Management (TM) approach of trusted third parties (such as any PKI CA ${ }^{1}$ ), and use the certification provided by them to assign roles to users. We derive our motivation for doing that from the review of traditional access control schemes that have adopted either an identity or capability-based approach to authorize users [Author-X thru XACL]. Such mechanisms do not scale well to the distributed Web services architecture, and hence would cause a significant burden to be attached to the enforcement of the access control scheme. This is because each credential needs an explicit delegation act by the respective domain administrators. In order to overcome this limitation, we outline a mechanism to incorporate trust in X-GTRBAC model in Section 3. In particular, we would use TM credentials (i.e. certificates) to allow trust establishment amongst distributed domains.

The remainder of this paper is organized as follows. We begin by providing a compendium of related work in the area of Web services security, and discuss how our framework aligns with the existing security architectures. We also review the features provided by existing Web-based access control schemes, and their suitability to Web services. We next introduce our trust-based context-aware access control model, which is based on a temporal extension of X-RBAC with trust domains incorporated into it. The paper concludes with the discussion of implementation architecture of our model and an overview of future research goals.

\section{Background and Related Work}

We shall now provide a background and compendium of current state of the art in Web services security. A fair amount of related research in this area is due to the industry, with standards such as Security Assertion Markup Language (SAML) [SAML] and eXtensible Access Control Markup Language (XACML) [XACML] being recently adopted. SAML defines an XML framework for exchanging authentication and authorization information

\footnotetext{
${ }^{1}$ Public Key Encryption Certification Authority
} 
for securing Web services, and relies on third-party authorities for provision of "assertions" containing such information. XACML is an XML framework for specifying access control policies for Web-based resources, and with significant extensions can potentially be applied to secure Web services. The XACML specification supports identity-based access control and incorporates some contextual information, such as location and time, into access decisions, without any formal context-aware access control model. There also are other emerging specifications, most notable amongst them are the ones outlined in WS security roadmap [WS Roadmap]. The road map consists of a bunch of component specifications, the core amongst them are WS-Security, WS-Policy, and WS-Trust. WS-Security is similar in intent and purpose to SAML, only uses a different technology. WS-Policy is used to describe the security policies in terms of their characteristics and supported features (such as required "security tokens", encryption algorithms, privacy rules, etc.). WS-Trust defines a trust model that allows for exchange of such security tokens (using mechanisms provided by WS-Security) in order to enable the issuance and dissemination of credentials within different trust domains, and establish online trust relationships. The models proposed in the roadmap have been directed primarily at authentication aspect of Web services security, with an emphasis on designing secure messaging protocols to communicate the security-relevant information, such as security tokens and characteristics of security policy. The specification leaves room for custom authorization models to be tied in to the architecture at the appropriate (i.e. WS-Policy) level. In this paper, we intend to present an access control model that is capable of doing exactly that; our XML-based framework allows easy integration into the existing XML-based architectures for Web services security, while providing an effective authorization mechanism suitable for Web services environment.

There has been an effort in the research community to highlight the challenges in Webbased access control within the XML framework, including both the initial DTD-based solutions [Bertino, Damiani, Vuong, XACL], and the more recent schema-based approaches [X-RBAC, Sandhu]. In [X-RBAC], we have presented X-RBAC, an XMLbased RBAC policy specification framework for enforcing access control in dynamic XML-based Web services. X-RBAC was designed to readily integrate within the XML framework, and emphasized simple, yet effective, administration through the use of RBAC. We also maintained that X-RBAC includes a comprehensive set of features that is comparable to the related access control schemes cited above, and is targeted for the Web services environment. Although X-RBAC and related schemes provide viable solutions, there remain issues that impede the development of effective access control models for Web services environment. Amongst them are the lack of context-aware models for access control, and reliance on identity or capability-based access control schemes. We next elaborate upon these issues, and propose an extended and trustenhanced version of our X-RBAC model in an attempt to address them.

\section{Trust-Enhanced X-GTRBAC Model}

This section begins with an introduction to the X-GTRBAC model. It then describes the mechanism to configure X-GTRBAC to provide context-aware trust-based access control in Web services. 


\subsection{X-GTRBAC- An Introduction}

The X-GTRBAC framework is based on Generalized Temporal Role Based Access Control (GTRBAC) model [GTRBAC]. X-GTRBAC augments GTRBAC with XML to allow for supporting the policy enforcement in a heterogeneous, distributed environment. GTRBAC extends the widely accepted Role Based Access Control (RBAC) model proposed in the NIST RBAC standard [NIST_RBAC]. RBAC uses the concept of roles to embody a collection of permissions within an organizational setup. Permissions are associated with roles through a permission-to-role assignment, and the users are granted access to resources through a user-to-role assignment [RBAC96]. GTRBAC provides a generalized mechanism to express a diverse set of fine-grained temporal constraints on user-to-role and permission-to-role assignments in order to meet the dynamic access control requirements of an enterprise. X-GTRBAC allows specification of all the elements of the GTRBAC model. These specifications are captured through a contextfree grammar called X-Grammar, which follows the same notion of terminals and nonterminals as in BNF, but supports the tagging notation of XML that also allows expressing attributes within element tags. The detailed specification of these elements of X-GTRBAC framework can be found in [X-GTRBAC]. Table 1 below enlists the salient features of the model.

Table 1. Salient Features of X-GTRBAC

\begin{tabular}{|c|c|c|}
\hline Element Type & Element Name & Purpose \\
\hline \multirow[t]{3}{*}{ RBAC Element } & XML User Sheet (XUS) & $\begin{array}{l}\text { Declares the users and their authorization } \\
\text { credentials }\end{array}$ \\
\hline & XML Role Sheet (XRS) & $\begin{array}{l}\text { Declares the roles, their attributes, role hierarchy, } \\
\text { and any separation of duty and temporal constraints } \\
\text { associated with roles }\end{array}$ \\
\hline & XML Permission Sheet (XPS) & Declares the available permissions \\
\hline \multirow[t]{2}{*}{$\begin{array}{l}\text { RBAC } \\
\text { Assignments }\end{array}$} & $\begin{array}{l}\text { XML User-to-Role Assignment } \\
\text { Sheet (XURAS) }\end{array}$ & $\begin{array}{l}\text { Defines the rules for assignment of users to roles; } \\
\text { these assignments may have associated temporal } \\
\text { constraints }\end{array}$ \\
\hline & $\begin{array}{l}\text { XML Permission-to-Role } \\
\text { Assignment Sheet (XPRAS) }\end{array}$ & $\begin{array}{l}\text { Defines the rules for assignment of permissions to } \\
\text { roles; these assignments may have associated } \\
\text { temporal constraints }\end{array}$ \\
\hline $\begin{array}{l}\text { RBAC } \\
\text { Constraints }\end{array}$ & $\begin{array}{l}\text { XML Separation Of Duty } \\
\text { Definition Sheet (XSoDDef) }\end{array}$ & Defines the separation of duty constraints on roles \\
\hline \multirow[t]{2}{*}{$\begin{array}{l}\text { GTRBAC } \\
\text { Constraints }\end{array}$} & $\begin{array}{l}\text { XML Temporal Constraint } \\
\text { Definition Sheet } \\
\text { (XTempConstDef) }\end{array}$ & $\begin{array}{l}\text { Defines the temporal constraints on role enabling } \\
\text { and activation; also defines temporal constraints for } \\
\text { user-to-role and permission-to-role assignments }\end{array}$ \\
\hline & $\begin{array}{l}\text { XML Trigger Definition Sheet } \\
\text { (XTrigDef) }\end{array}$ & $\begin{array}{l}\text { Defines context-based triggers for invocation of } \\
\text { periodic events subject to associated constraint } \\
\text { evaluation }\end{array}$ \\
\hline $\begin{array}{l}\text { Authorization } \\
\text { Credentials }\end{array}$ & $\begin{array}{l}\text { XML Credential Type } \\
\text { Definition Sheet } \\
\text { (XCredTypeDef) }\end{array}$ & Defines the available credential types \\
\hline
\end{tabular}


We now describe the mechanism to configure X-GTRBAC to provide context-aware trust-based access control in Web services. Toward that end, we need to outline a set of formal specifications to capture contextual information, and illustrate how it can be incorporated within the access control model. In addition, we would need to provide an interface to the system to accept TM credentials instead of its usual user credentials as the basis of privilege assignments.

\subsection{Context-aware access control}

This section defines the set of specifications needed to configure X-GTRBAC for context-aware access control in Web services environment. We base our set of specifications on a tuple language that can be readily mapped to our existing XML-based framework. In the following, we provide the formal definition of context, and then use that to provide the definition for a service_access_request. In order to formalize the context, we introduce a type system to allow specifying domains of legal values for various context parameters. Our formal model relies on the components we define below:

Parameter Name Set: A set PN to denote the possible names of context parameters

Parameter Type Set: A set PT to denote the possible types of context parameters

Context Parameter: A context parameter is represented by a data structure $\mathrm{p}$, having the following fields: name $\in \mathrm{PN}$, type $\in \mathrm{PT}$, and a function getValue () .

Roles Set: $\quad \mathrm{RR}=\left\{\mathrm{rr}_{1}, \ldots, \mathrm{rr}_{\mathrm{k}}\right\}$, where $\mathbf{r r}_{\mathrm{i}}, 1 \leq \mathrm{i} \leq \mathrm{k}$ is a regular role in GTRBAC

Operations Set: $\mathrm{RO}=\left\{\mathrm{ro}_{1}, \ldots, \mathrm{ro}_{\mathrm{k}}\right\}$, where $\mathbf{r o}_{\mathrm{k}}, 1 \leq \mathrm{i} \leq \mathrm{k}$ is a regular operation in GTRBAC

Service: $\quad$ A service is an abstraction of the operations provided by the system on its resources. Formally, a service is a subset of the data set $\mathrm{RO}$, and is designated by the service name srv that is defined per the wsdl:service element of the Web Services Description Language (WSDL) document

Services Set: $\quad \operatorname{SRVS}=\left\{\operatorname{srv}_{1}, \ldots, \operatorname{srv}_{\mathrm{k}}\right\}$, where $\operatorname{srv}_{\mathrm{i}}, 1 \leq \mathrm{i} \leq \mathrm{k}$ is a service.

Definition 1: (Context): A context set $C$ consists of $n$ context parameters $\left\{p_{1}, \ldots, p_{n}\right\}, n \geq 0$, s.t. for any $p_{i}, p_{j}$, with $i \neq j$ and $1 \leq i, j \leq n$, we have that $p_{i}$. name $\neq p_{j}$. name (i.e. the parameter names must be distinct).

We mention here that PN and PT constitute a set of pre-specified parameter names and types determined by the SSO. For example, the set PN may be defined as: PN $=\{$ time_of_day, location, duration, system_load $\}$, with the corresponding set PT defined as: $\mathrm{PT}=\{$ Time, String, Long, Integer $\}$. The p.getValue() function is used to dynamically compute the value of the parameter, and its implementation is system-dependent. For built-in system parameters, such as time_of_day, it might just serve as a wrapper around system functions such as getCurrentTime(). The dynamic mechanism to compute parameter values especially helps in the case of mobile users accessing Web-based services, because in such environments the parameter values are constantly changing and may need to be re-evaluated at certain intervals. Additionally, for dynamic access constraints, such as duration, getvalue () would be called periodically to ensure that the constraint is always satisfied. We also note from the preceding definition that the context may be an empty set. 
Definition 2: (Service_access_request): A service access request is defined as a triple $<$ role, srv, context> where role $\in \mathrm{RR}$, $\mathrm{srv} \in \mathrm{SRVS}$, and context is defined according to Definition 1 and captured dynamically at the time of the access request.

Based on the service_access_request, the system determines the applicable access policy for the requested service. This policy will be based on a set of constraints on the role and service name, and evaluated in conjunction with the available contextual information to enforce fine grained access control. An access policy consists of a collection of access conditions. In order to formulate an access condition, we refer to the notion of parameterized roles of [X-GTRBAC]. Parameterized roles are roles supplemented with role_attributes. The attributes set $\mathrm{A}$ of a role contains a collection of contextual attributes (such as time, location or system load) that may be used to define context-based conditions on roles. The values of these attributes are specified by the SSO, and these values are compared with the values of the supplied context parameters in order to evaluate an access request. The set of contextual attributes of a role is hence a subset of the set $\mathrm{C}$ of context parameters, and follows the same type system. We formally define the access_policy below. We assume the existence of a function getAttributesset(role), which returns the set A for a given role.

Definition 3: (Access Policy): Let $r \in \mathrm{RR}$ be a role, srv $\in$ SRVS be a service name denoting a service. The access policy AP for a $(r, s r v)$ pair is a set of clauses, where each clause is a Boolean combination of expressions. An expression is of the form <attr $\Theta$ val $>$ where attr is a role attribute s.t. attr $\in$ getAttributesset $(r)$, val is the value of the parameter as specified by the SSO in order for role $r$ to access the service srv, and $\Theta$ is any relational/comparison operator.

It may be mentioned that we have intentionally kept our model generic enough, as it is unlikely for any one model to capture all types of contextual information and associated conditions that might arise in practice. But for most practical purposes, the sets PN, PT and role_attributes may be extended according to the system requirements in order to define access conditions based on appropriate context parameters.

We now give the following set of algorithms to evaluate a service_access_request.

\begin{tabular}{|c|c|}
\hline 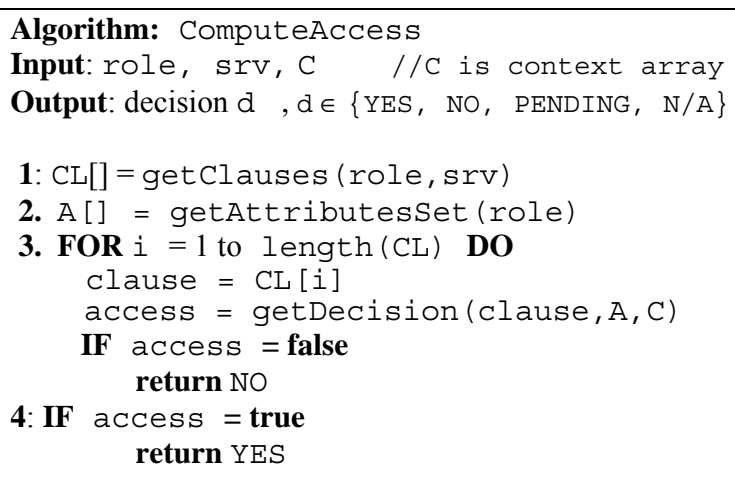 & $\begin{array}{l}\text { Algorithm: getDecision } \\
\text { Input: clause, } A, C \\
\text { Output: result //boolean } \\
\text { 1. initizalize(result []) } \\
\text { 2. FOR } i=1 \text { to size(clause) DO } \\
\quad \text { expr }=\text { clause.getExpr (i) } \\
\quad \text { result }[i]=\text { evaluateExp (expr, A, C) } \\
\text { 3. return computeResult (result []) }\end{array}$ \\
\hline
\end{tabular}




\begin{tabular}{|c|c|}
\hline $\begin{array}{l}\text { Algorithm: EvaluateExpression } \\
\text { Input: expr, A, C } \\
\text { Output: result } \\
\text { 1/boolean } \\
\text { 1. name = expr.getAttrName () } \\
\text { 2. attr = getAttribute (A, name) } \\
\text { 3. result = checkCondition(attr, C) } \\
\text { 4. return result }\end{array}$ & $\begin{array}{l}\text { Algorithm: checkCondition } \\
\text { Input: attr, } \mathrm{C} \\
\text { Output: result } \\
\text { 1. } \mathrm{p}=\text { match }(\mathrm{C} \text {, attr }) \\
\text { IF } \mathrm{p} \cdot \operatorname{getValue}() \Theta \mathrm{val} \\
\text { result = true } \\
\text { ELSE } \\
\quad \text { result = false } \\
\text { 2.: return result }\end{array}$ \\
\hline
\end{tabular}

The ComputeAccess algorithm works as follows. In Step 1, the clauses corresponding to the (role, srv) pair are retrieved from the AP into a dynamic array CL. Step 2 retrieves the attributes of the role into a dynamic array A. In Step 3, the algorithm loops over the array CL and calls the routine getDecision() for each of the clauses. Each clause has potentially multiple expressions, and so each expression is evaluated using the evaluateExpr () routine. For each expression, this routine retrieves the attribute from the attribute array $\mathrm{A}$ and then calls the routine checkCondition() to evaluate the conditions corresponding to this role attribute. This routine loops over the set $\mathrm{c}$ of supplied context parameters and finds the matching context parameter for this role attribute by calling the match () routine, which internally compares the name and type of the two entities. Since the set A is a subset of set c, this search always results in a match. When a match is found, it compares their values according to the operator specified in the AP. If the condition is satisfied, a value of true is returned to getDecision. After the result for all access conditions within the clause has been computed, the getDecision routine then computes the overall result for the clause and returns it to ComputeAccess. If any of the clauses evaluates to false, a NO is returned as the output of the ComputeAccess algorithm, because the overall access decision is a conjunction of all individual clauses. Otherwise, after the loop terminates successfully over all the clauses, a YES is returned. Other decisions such as PENDING or N/A may also be returned by incorporating system-specific logic into the algorithm.

As an illustration, consider the example of a recently launched initiative of a German insurance company [Example1]. The company leverages Web services technology to introduce online visitors to its services, and allows them to purchase insurance coverage through an entirely digital process. The evaluation of an online coverage request requires several kinds of personal information to be made available, and the same needs to persist in the company's database for a subsequent evaluation of an insurance claim. At that point, however, the access to the customer's resources should only be granted after establishing the fact that the requestor indeed is "the" genuine customer. For instance, assume that the following service_access_request is submitted for evaluation to the system:

$$
\begin{aligned}
& <\text { role=priv_cust, service_name='review_claim", context }=\left\{\mathrm{p}_{1}\{\mathrm{time}, 12 \mathrm{PM}\},\right. \\
& \mathrm{p}_{2}\{\text { location, "WashDC" }\}, \mathrm{p}_{3}\{\text { duration, } 0\}, \mathrm{p}_{4}\{\text { system_load, "low" }\}>.
\end{aligned}
$$

This request says that a user belonging to the priv_cust (privileged customer) role has requested to review an online insurance claim through the Web-based review_claim service offered by the company. The context recorded at the time of access request is 
provided to the system as part of the request. Note that duration is initialized to 0 because the access has not yet started. Now, assume that the following AP is applicable to the given (role, srv) pair:

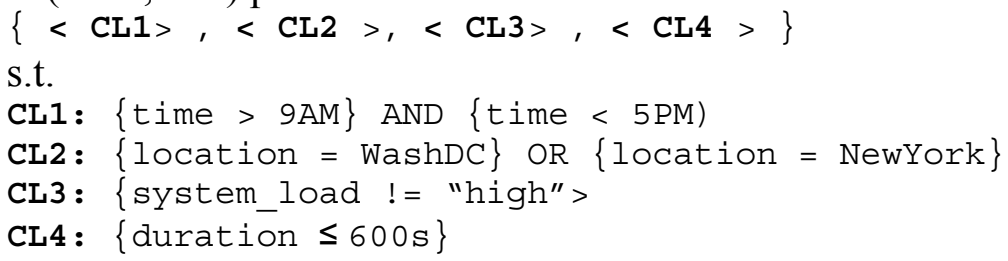

Based on this information, the system would return an authorization decision for this service_access_request. The available contextual information indicates that the access conditions are satisfied. In addition, due to the duration constraint specified for the requested service and enforced by the dynamic temporal constraint mechanism of GTRBAC, the access duration of the user is continuously monitored, and any violation thereof is detected on a per-user basis by the GTRBAC Processor (see Figure 1). The mechanism to deal with the violation is system-specific, but GTRBAC allows a trigger mechanism to take immediate actions in such situations (such as de-activating the role for the given user). Detailed discussion on such mechanisms can be found in [X-GTRBAC].

\subsection{Incorporating trust domains}

In this section, we briefly describe a mechanism to incorporate trust domains in $\mathrm{X}$ GTRBAC to enable effective access control in a distributed environment, where user identities are not known a-priori. Since X-GTRBAC makes the access decisions based on the eligible roles for known users, we can use TM credentials to assign roles to users. While it is sometimes viewed as appropriate in TM to adopt a direct authorization model, i.e. to combine authentication and access control into one authorization step [Bla99a], we would like to motivate here that the indirection through roles helps scalability and flexibility in the case of large scale open systems, especially Web services. Hence, a significant advantage that accompanies the role-based approach adopted in our framework is that of simplified authorization administration [RBAC96]. An earlier approach that merged features from $\mathrm{TM}$ and $\mathrm{RBAC}$, called the Role based Trust management framework (RT), was reported in [Li02a]. However, our primary goals are different from RT. The latter is primarily a TM credential exchange and distribution mechanism to assist authorizations in a distributed environment; it does not support an elaborate access control scheme beyond the basic permission-to-role assignment mechanism in RBAC. We focus on providing a context-aware access control model for the Web services environment, and rely on TM credentials for determining the trust level (i.e. role) associated with a user. As mentioned in the introduction, the trust level can be subsequently adjusted based on the user's activity profile. Such a profile can be maintained by logging the contextual information associated with the invocation and acceptance of a service_access_request.

The use of TM credentials to establish role memberships of users requires the XGTRBAC model to be adapted to accept distributed TM credentials. We touch upon the mechanisms needed to do this in the next section, but leave an elaborate treatment of the same for some future work, as it is not the focus of our current paper. It may be noted 
here that the trust-based approach to verifying user credentials effectively adds authentication support to our existing authorization model.

\section{Implementation Architecture}

There is an on-going effort underway on extending our implementation prototype, first reported in [X-RBAC]. Figure 1 illustrates the architecture and design of the extended version of the X-GTRBAC prototype, incorporating context-aware access control.

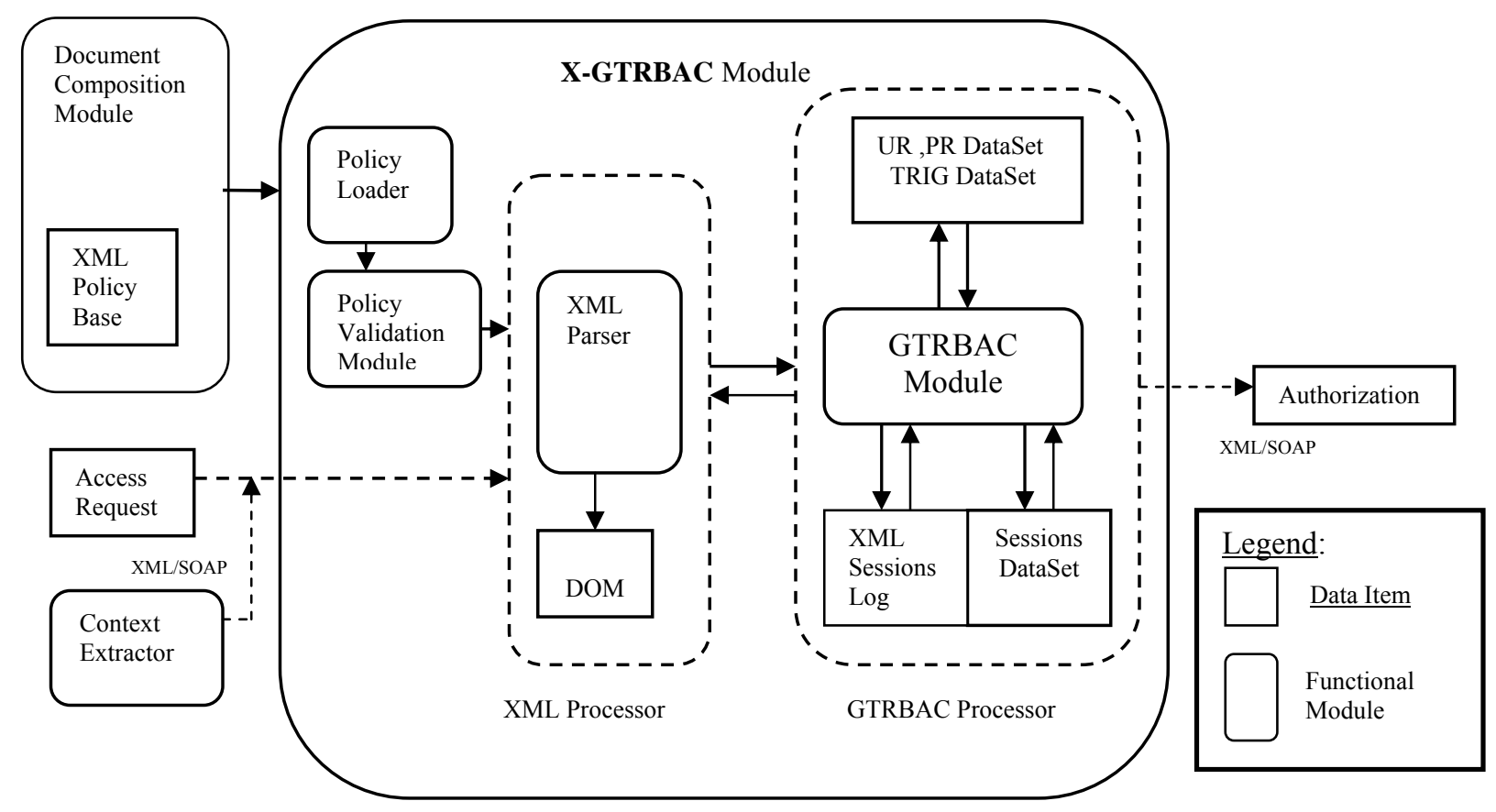

Figure 1: Context Aware X-GTRBAC System Architecture

The major system components depicted in the figure are summarized in Table 2. The existing prototype incorporates the temporal constraint enforcement mechanism as per the GTRBAC model. The generalization of the contextual information to include parameters other than time as described in the paper is being incorporated into the system. We are also working toward a set of specifications that would allow us to substitute the existing credential evaluation mechanism with that involving TM credentials. Because of the modular design of X-GTRBAC, this task can be accomplished with only slight modifications in the overall architecture. The components affected would be (i) the XML Policy Base, since it would now need to store a different XCredTypeDef sheet based on TM credentials, and (ii) the XML Processor, since it would now employ a different evaluation logic for processing credential declarations. Our set of specifications would be XML-based, and hence can be expected to integrate well with the existing framework. 
Table 2. Description of X-GTRBAC system modules

\begin{tabular}{|l|l|}
\hline \multicolumn{1}{|c|}{ Module Name } & \multicolumn{1}{c|}{ Description } \\
\hline Document Composition Module (DCM) & $\begin{array}{l}\text { Used to compose the policy documents; contains the XML- } \\
\text { Policy Base that serves as the document repository; is an } \\
\text { external component of the model }\end{array}$ \\
\hline Policy Loader & $\begin{array}{l}\text { The interface of the system to the DCM; used to load the } \\
\text { XML policy files into the system }\end{array}$ \\
\hline Policy Validation Module & $\begin{array}{l}\text { Validates the XML policy files for existence checking and } \\
\text { type conformance according to policy rules; XML syntax } \\
\text { validation is also implicitly done }\end{array}$ \\
\hline XML processor & $\begin{array}{l}\text { Contains an XML parser that generates the DOM tree } \\
\text { representation of the XML policy files }\end{array}$ \\
\hline GTRBAC Processor & $\begin{array}{l}\text { Contains a GTRBAC Module that translates the DOM tree } \\
\text { representation to internal RBAC data structures representing } \\
\text { the system state at any time; maintains logs of sessions and } \\
\text { updates the system data structures to allow contextual } \\
\text { information to be incorporated in access decisions }\end{array}$ \\
\hline
\end{tabular}

\section{Conclusion}

In this paper, we have outlined a mechanism to develop a trust-based, context-aware access control model for Web services based on the X-GTRBAC framework. XGTRBAC is a temporal extension of the earlier X-RBAC model for access control in Web services. The mechanism presented in the paper extends X-GTRBAC to support context-aware access control based on both temporal and non-temporal contextual conditions. In addition, we outline a mechanism to incorporate trust domains into XGTRABC by the use of distributed TM credentials for unknown users. Such an approach effectively adds authentication support to our system. We have discussed the configuration of X-GTRBAC for its application in Web services environments, and also proposed extensions to our current implementation architecture for the purposes outlined in this paper. We intend to report the detailed results of our on-going implementation efforts in some future work. We also plan to explore a mechanism to allow specification of certain role attributes whose values are not known a-priori, and may be dynamically computed based on runtime information. This scheme is particularly useful where the same role may have a different set of associated privileges depending on the value of the role attributes. Another future direction of research would be to investigate the suitability of the proposed administration model for X-GTRBAC [X-GTRBAC-Admin] to Web services. We expect to see our framework evolve with time, as Web services standards are continually being enhanced, and would likely incorporate additional security mechanisms, such as secure messaging and transaction support, into our system leveraging the power of existing and emerging Web services specifications.

\section{References}

[Example1] Accenture Web Services Case Study http://www.accenture.com/xd/xd.asp?it=enweb\&xd=services $\backslash$ microsoft $\backslash$ case $\backslash$ micr ergo.xml 
[Author-X] E. Bertino, S. Castano, E. Ferrari, "Securing XML Documents with Author X", IEEE Internet Computing May-June 2001.

[Damiani] E. Damiani, S. D. C. di Vimercati, S. Paraboschi, P. Samarati, "A Fine Grained Access Control System for XML Documents”, ACM Transactions on Information and System Security, Volume 5, Issue 2, May 2002.

[Sandhu] X. Zhang, J. Park and R. Sandhu, Schema based XML Security: RBAC Approach, IFIP WG 11.32003.

[Vuong] N. N. Vuong, G. S. Smith, Y. Deng, "Managing Security Policies in a Distributed Environment Using eXtensible Markup Language (XML)”, Symposium on Applied Computing, March 2001

[X-RBAC] R. Bhatti, J. B. D. Joshi, E. Bertino, A. Ghafoor, “Access Control in Dynamic XML-based Web-Services with X-RBAC”, In proceedings of The First International Conference on Web Services, Las Vegas, June 23-26, 2003.

[XACL] S. Hada, M. Kudo, "XML Access Control Language: Provisional Authorization for XML Documents", October 16, 2000, Tokyo Research Laboratory, IBM Research.

[NIST_RBAC] D. F. Ferraiolo, R. Sandhu, S. Gavrila, D. Richard Kuhn, Ramaswamy Chandramouli, "Proposed NIST standard for role-based access control", ACM Transactions on Information and System Security (TISSEC), Volume 4 , Issue 3 (August 2001).

[RBAC96] R. Sandhu, E. J. Coyne, H. L. Feinstein, C. E. Youman, "Role Based Access Control Models", IEEE Computer Vol. 29, No 2, February 1996.

[GTRBAC] J. B. D. Joshi, Elisa Bertino, Usman Latif, Arif Ghafoor, "Generalized Temporal Role Based Access Control Model (GTRBAC) (Part I) - Specification and Modeling", Submitted to IEEE Transaction on Knowledge and Data Engineering. Available as technical report at: https://www.cerias.purdue.edu/tools_and_resources/bibtex_archive/archive/2001-47.pdf

[X-GTRBAC] R. Bhatti, "X-GTRBAC: An XML-based Policy Specification Framework and Architecture for Enterprise-Wide Access Control", Masters thesis, Purdue University, May 2003. Available as technical report at: https://www.cerias.purdue.edu/tools and resources/bibtex archive/archive/2003-27.pdf

[X-GTRBAC-Admin] R. Bhatti, J. B. D. Joshi, E. Bertino, A. Ghafoor, "X-GTRBAC Admin: A Decentralized Administration Model for Enterprise Wide Access Control", Available as technical report at: https://www.cerias.purdue.edu/tools_and_resources/bibtex_archive/archive/2004-04.pdf

[WS Roadmap] Security in a Web Services World: A Proposed Architecture and Roadmap http://www-106.ibm.com/developerworks/security/library/ws-secmap/

[SAML] OASIS, Security Services TC, http://www.oasis-open.org/committees/tc_home.php?wg_abbrev=security

[XACML] XACML 1.0 Specification http://xml.coverpages.org/ni2003-02-11-a.html

[Bla99a] M. Blaze, J. Feigenbaum, J. Ioannidis, and A. D. Keromytis. The KeyNote Trust Management System, version 2. IETF RFC 2704, September 1999.

[Li02a] N. Li, J..C. Mitchell, and W. H. Winsborough. Design of a role-based trust management framework. In Proceedings of the 2002 IEEE Symposium on Security and Privacy. IEEE Computer Society Press, May 2002. 